\title{
A robot-based gait training therapy for pediatric population with cerebral palsy: goal setting, proposal and preliminary clinical implementation
}

Cristina Bayón ${ }^{1}$, Teresa Martín-Lorenzo ${ }^{2,3}$, Beatriz Moral-Saiz 2,4, Óscar Ramírez ' Álvaro Pérez-Somarriba², Sergio Lerma-Lara ${ }^{2,4}$, Ignacio Martínez ${ }^{2}$ and Eduardo Rocon ${ }^{1,5^{*}}$

\begin{abstract}
Background: The use of robotic trainers has increased with the aim of improving gait function in patients with limitations. Nevertheless, there is an absence of studies that deeply describe detailed guidelines of how to correctly implement robot-based treatments for gait rehabilitation. This contribution proposes an accurate robot-based training program for gait rehabilitation of pediatric population with Cerebral Palsy (CP).

Methods: The program is focused on the achievement of some specifications defined by the International Classification of Functioning, Disability and Health framework, Children and Youth version (ICF-CY). It is framed on 16 non-consecutive sessions where motor control, strength and power exercises of lower limbs are performed in parallel with a postural control strategy. A clinical evaluation with four pediatric patients with CP using the CPWalker robotic platform is presented.

Results: The preliminary evaluation with patients with CP shows improvements in several aspects as strength $(74.03 \pm 40.20 \%)$, mean velocity $(21.46 \pm 33.79 \%)$, step length $(17.95 \pm 20.45 \%)$ or gait performance (e.g. $66 \pm 63.54 \%$ in Gross Motor Function Measure-88 items, E and D dimensions).

Conclusions: The improvements achieved in the short term show the importance of working strength and power functions meanwhile over-ground training with postural control. This research could serve as preliminary support for future clinical implementations in any robotic device.

Trial registration: The study was carried out with the number R-0032/12 from Local Ethical Committee of the Hospital Infantil Niño Jesús. Public trial registered on March 23, 2017: ISRCTN18254257.
\end{abstract}

Keywords: Robotic training, Therapy, Cerebral palsy, Exoskeleton, Rehabilitation, Gait

\section{Background}

Gait limitation is one of the main impairments in children with Cerebral Palsy (CP) [1]. This mobility deficiency in $\mathrm{CP}$ is commonly the consequence of a damage of the child's Central Nervous System (CNS), and an optimal functional training is required in order to maximize the

\footnotetext{
* Correspondence: e.rocon@csic.es

${ }^{1}$ Centre for Automation and Robotics (CAR), CSIC-UPM, Ctra Campo Real km

0.2 - La Poveda-Arganda del Rey, 28500 Madrid, Spain

${ }^{5}$ Universidade Federal do Espírito Santo Vitória, Brazil

Full list of author information is available at the end of the article
}

improvements [2], which will highly contribute to the enhancement of the independence and, therefore, the quality of life for both the young patient and his family [3].

The use of robotic trainers for neurorehabilitation applications has increased in the last decades, both in adulthood and childhood, and in several motor diseases [3-5]. Robot-based therapies have been developed and improved beyond reducing the clinician's effort. Currently, a new generation of robotic devices [6-8] provides means for encouraging the patients to an active participation in exercises, which are now more task specific. Both the implemented novel control strategies and

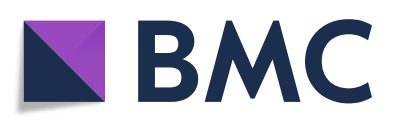

(c) The Author(s). 2018 Open Access This article is distributed under the terms of the Creative Commons Attribution 4.0 International License (http://creativecommons.org/licenses/by/4.0/), which permits unrestricted use, distribution, and reproduction in any medium, provided you give appropriate credit to the original author(s) and the source, provide a link to the Creative Commons license, and indicate if changes were made. The Creative Commons Public Domain Dedication waiver (http://creativecommons.org/publicdomain/zero/1.0/) applies to the data made available in this article, unless otherwise stated. 
the modularity of new exoskeletons and gait trainers offer promising possibilities to enhance the rehabilitation outcomes by adapting the treatment to the patient's needs $[9,10]$. Nevertheless, so far there is not enough evidence to ensure that classic robot-based rehabilitation provides better treatment outcomes by itself than conventional physical strategies in childhood [11]. New approaches are needed in order to improve the rehabilitation, making the robotic therapy a key feature of the change.

One of the main drawbacks for the everyday use of these technologies into the rehabilitation practice, apart from the price of these devices, is the absence of studies that describe a detailed robotic training program for gait rehabilitation. The wide variety of changes that could be applied to the parameters of robotic training therapies, makes unclear how to specify rehabilitation settings with the aim of providing a suitable solution for a large population size. Additionally, most of current studies are only focused on lower limbs strategies. However, the upper body (head and trunk movement) also influences gait function through walking balance [12], so a proper program should not ignore these features.

This manuscript presents a detailed robot-based therapy proposal for the rehabilitation of gait function in children with $\mathrm{CP}$, which is based on the achievement of some specifications defined by the International Classification of Functioning, Disability and Health framework, Children and Youth version (ICF-CY) [13]. It contributes with better answers on how to implement robotic rehabilitation following defined guidance, establishing the baseline settings and subsequently tailoring the therapy to each patient.

The proposed robotic rehabilitation therapy works around a key factor: the implementation of strength and power exercises at the same time than over-ground walking guidance, performing in parallel an active head-trunk control therapy. As a result, the robot-based program recreates a situation as similar as possible to a real gait scenario, and encourages the patients to control different movements associated with gait: not only individual movements of lower limb joints but also the synergy between them while maintaining a proper posture of upper body. We hypothesize that these essential components, performed following an appropriate progression of the variables, will boost the rehabilitation of our patients.

Eventually, the robot-based therapy proposed in this manuscript is evaluated in four patients with $\mathrm{CP}$ in order to provide preliminary results of its application. Although the proposed robot-based treatment could be implemented in any robotic device adapted for that, in this study we selected the CPWalker training platform [7] as one scenario to test the effectiveness of the approach. This device is a novel robotic prototype with partial body weight support (PBWS) for over-ground rehabilitation of children with CP. It provides means for adapting the therapy to the user's necessities through different levels of assistance in multi-joint over-ground training. Other gait trainers, such as Lokomat [14] or LOPES [6], could have been also used to evaluate this training method, since they are also able to tailor each therapy session to the patient's needs.

\section{Methods}

\section{Rehabilitation device}

The CPWalker rehabilitation platform [7] is a robotic device composed by an exoskeleton linked to a walker that provides support and balance to the child during over-ground training (Fig. 1). The device is able to implement users' PBWS and allows the adaptation of exercises to the patient's capabilities by means of individual controllers for each joint, which increases the modularity of the system [7]. Each joint of the CPWalker exoskeleton can operate in a wide range of modes (see Fig. 1):

i) Position control mode: in this mode, the robot imposes a prescribed gait pattern to the user's lower limbs. The aim is that the patient learns the walking motion sequence correctly.

ii) Impedance control modes: these modes take into account the patients' collaboration. Thereby, the prescribed gait pattern should be achieved by the sum of robotic assistance and patient's cooperation. Three different modes of impedance may be executed in CPWalker (high, medium and low), which tolerate variable deviations from the programmed gait trajectories, enhancing the patients' participation and taking advantage of their residual movements through assist as needed strategies (AAN).

iii) Zero-force control mode: in this mode, the trajectory reference is not given, and the patient is who entirely moves the legs with a minimal resistance of the exoskeleton. It is used with patients with enough motor control (acquired with the previous modes) but poor balance, so the CPWalker provides stability and PBWS while the patient implements the gait pattern.

Within each mode, some variables of the robotic platform are updated along the treatment sessions: PBWS, gait velocity and the percentage of the total range of motion (ROM) [9]. The possibility of varying these parameters enables the customization of the therapy to the progression of each patient and gives a higher versatility for the treatment design. Additionally, the robotic platform also includes a biofeedback strategy to motivate the children to actively correct their posture during 


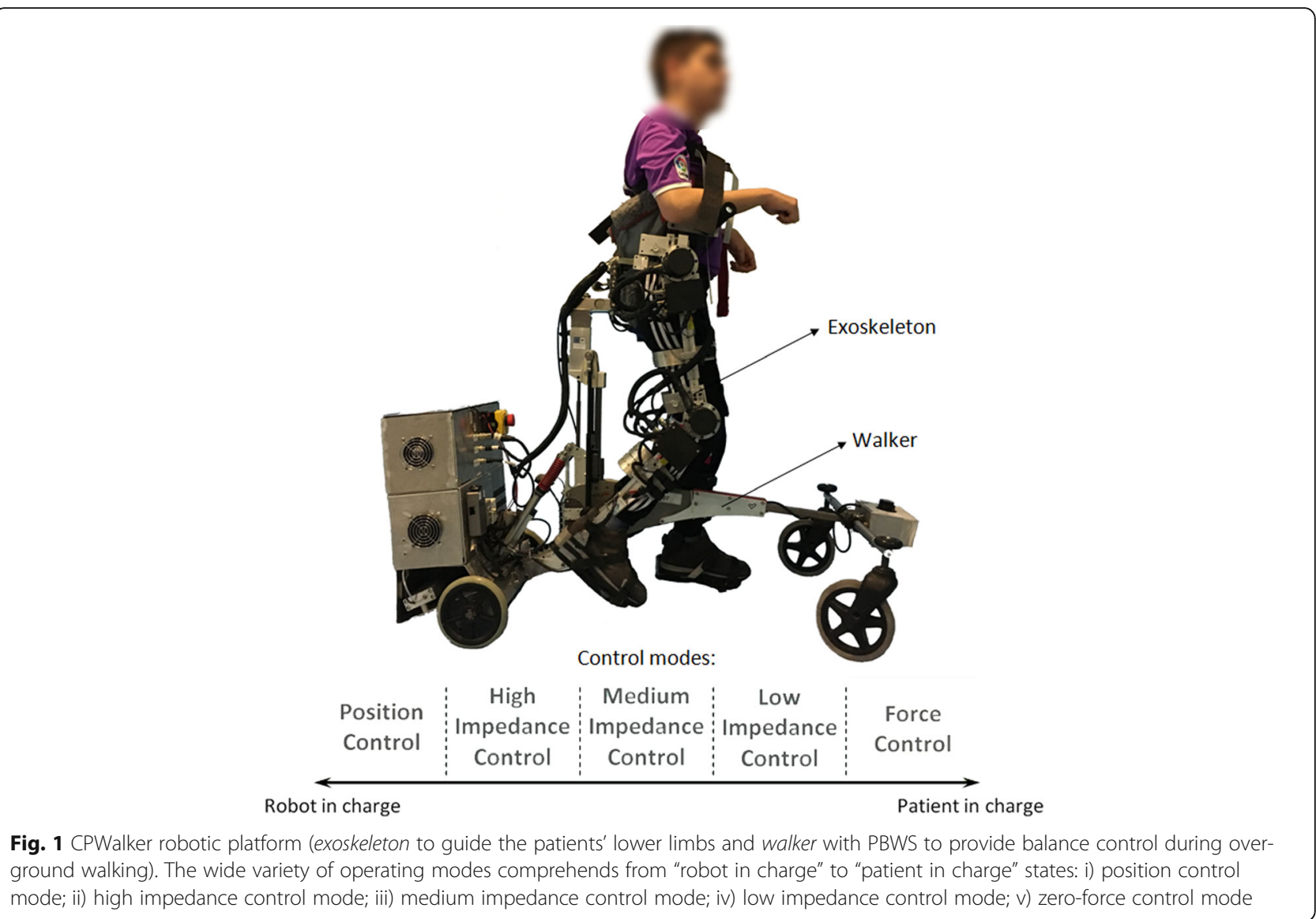

walking $[7,9]$. The CPWalker robotic platform may be easily controlled by a clinician through an intuitive interface, which controls and monitors the exercises in real time.

The variety of control modes, the number of adjustable parameters, the ease of setting the variables individually for each joint and the possibility of implementing different strategies associated with gait simultaneously, made CPWalker an appropriate platform for testing the robotic therapy proposed in this manuscript.

\section{Robotic training program}

In order to define the objectives of the robot-based treatment, the we adopted the conceptual framework of ICF-CY [13]. The proposal was focused on improving the principal gait-related functions derived from this international classification. Concretely, the selected goals of the ICF-CY to be achieved with the treatment and the work methodology implemented in the robotic device (in this case CPWalker), are presented in Table 1.

To achieve the goals presented in Table 1, we previously performed a systematic selection of variables based on the requirements of the National Strength and Conditioning Association (NSCA) youth training guidelines, which suggests that eccentric and explosive strength exercises should be the beginning of a proper training to ensure greater muscle power generation and the transference of gains to gait (Table 2)

According to the proposed objectives (Table 1) and complying with the NSCA youth training guidelines (Table 2), the treatment was conceptualized into two main phases, where the ROM, PBWS and gait velocity were the principal parameters under variation. The intention was that the patient maximized the gains acquired in the whole rehabilitation period (ideally the sum of robot-based exercises and common non-robotic therapy). A detailed description of each phase follows:

i) First phase: the main aim of this phase was to improve motor control, teaching the patients the correct sequence of motion and increasing strength. The patients were requested to follow the movements established by the exoskeleton with the minimal possible resistance during swing period, pushing the ground at each step and trying to keep the maximum flexion-extension values at the end of each gait cycle. Instructions were given to ensure the comprehension of normative gait patterns, and verbal encouragement in addition to direct feedback by graphics in real-time was delivered throughout the sessions. 
Table 1 Goal settings of the ICF-CY and the robot-based solutions adopted with CPWalker platform

\begin{tabular}{ll}
\hline ICF-CY functional domain [13] & Implementation on CPWalker \\
\hline $\begin{array}{l}\text { Mobility and stability of joint } \\
\text { functions (b710, b715) }\end{array}$ & $\begin{array}{l}\text { The different control modes of the exoskeleton are used to guide the movement of a single } \\
\text { or multiple joints, improving motor control. The exoskeleton also helps to maintain stability } \\
\text { through the coordinated actions of surrounding tissues. This domain is exercised along the } \\
\text { whole treatment with diverse robotic assistance. }\end{array}$ \\
& $\begin{array}{l}\text { The second phase of the training requires the patient to contract a muscle or muscle groups to } \\
\text { generate the necessary force in order to start and maintain the movement with AAN strategies. }\end{array}$ \\
& $\begin{array}{l}\text { The force must be maintained for a time in the extremes of the gait pattern (maximum flexion } \\
\text { and extension) in order to reach the these maximum values in the complete range of motion. }\end{array}$
\end{tabular}

Muscle endurance

functions (b740)

Control of voluntary movement functions (b760)

Gait pattern functions (b770)

Maintaining a body

position (d415)

Walking (d450)

Proprioceptive functions (b260)

Formal relationships (d740)
Muscle endurance is exercised when the patient is requested to sustain a muscle contraction to finalize the required movement with AAN strategies, mainly in the extremes of the gait pattern (maximum flexion and extension).

The voluntary movement is implemented through the control and coordination of simple and complex movements to collaborate with AAN strategies. Lower impedance implies more patient's control.

Motor control and gait pattern functions are trained through the different control modes to guide the lower limbs following prescribed gait patterns at several velocities and supports.

A biofeedback strategy for postural control is used to notify the patients when they lose the correct position of the upper body. See section "Postural control".

Over-ground walking training is executed in all sessions with controlled PBWS, at different velocities and supports.

During random moments of the first training session, the patients perceive feeling using a mask on the eyes at the same time than the robot performs the movement for single or multiple joints with $100 \%$ of PBWS.

Creating and maintaining patient-researcher relationship. See section "Motivation and inclusion of challenges". ii) Second phase: the main aim of this phase was to further train motor control and increase power in order to ensure the transference to the independent gait pattern. Aware of the sequence of movement of a normal gait pattern, the patient's contribution became an important aspect to develop neuroplasticity and preserve the gained motor control [15]. The active participation was achieved both by boosting the patient's motivation and by requiring self-activity [16]. The latter was implemented through AAN algorithms and the impedance control modes presented previously for CPWalker [7].

Figure 2 represents the schematic view of the therapy proposal. The treatment was composed of a total of 16 sessions (first phase: 8 sessions for strength training and motor control learning; and second phase: 8 sessions to transfer the gains to gait through power performance).

Table 2 National Strength and Conditioning Association (NSCA) youth training guidelines [32]

\begin{tabular}{lll}
\hline \multicolumn{2}{l}{ Adapted youth resistance training NSCA guidelines } \\
\hline Variables & Strength & Power \\
\hline Muscle actions & Eccentric / Concentric & Eccentric / Concentric \\
Exercise & Single / Multi-joint & Single / Multi-joint \\
Intensity & $\uparrow$ Load / $\downarrow$ Velocity & $\downarrow$ Load / $\uparrow$ Velocity \\
Velocity & Moderate & Moderate / Fast \\
\hline
\end{tabular}

Exercises were multi-joint and gait-oriented, demanding concentric-eccentric actions based on the gait phase that was being performed. During the whole treatment (first and second phases) the position of head and trunk during walking exercises were monitored, especially because these patients usually walked looking at the ground. For this purpose, the strategy for postural control of CPWalker encouraged the patients giving an acoustic feedback to when their position was inappropriate, so they could realize and rectified it by themselves. The program was reinforced with modifications on AAN levels according to the patient's evolution based on performance evaluations related to ROM, PBWS and gait velocity. Furthermore, several challenges were included to enhance the patients' motivation.

\section{Duration of the study}

The robot-aided treatment was proposed for a whole period of 2 monthly cycles (1 month for each phase of the treatment) with the aim of having enough sessions to generate significant neural changes [17]. The children trained 2 non-consecutive days per week for 8 weeks (16 sessions, see Fig. 2). The sessions consisted of a 10 $15 \mathrm{~min}$ warm-up and $60 \mathrm{~min}$ of over-ground exercise with CPWalker, including 3 min of independent gait as a cool-down phase.

As Fig. 2 indicates, the first 8 sessions corresponded with general motor control and strength exercises, where the 


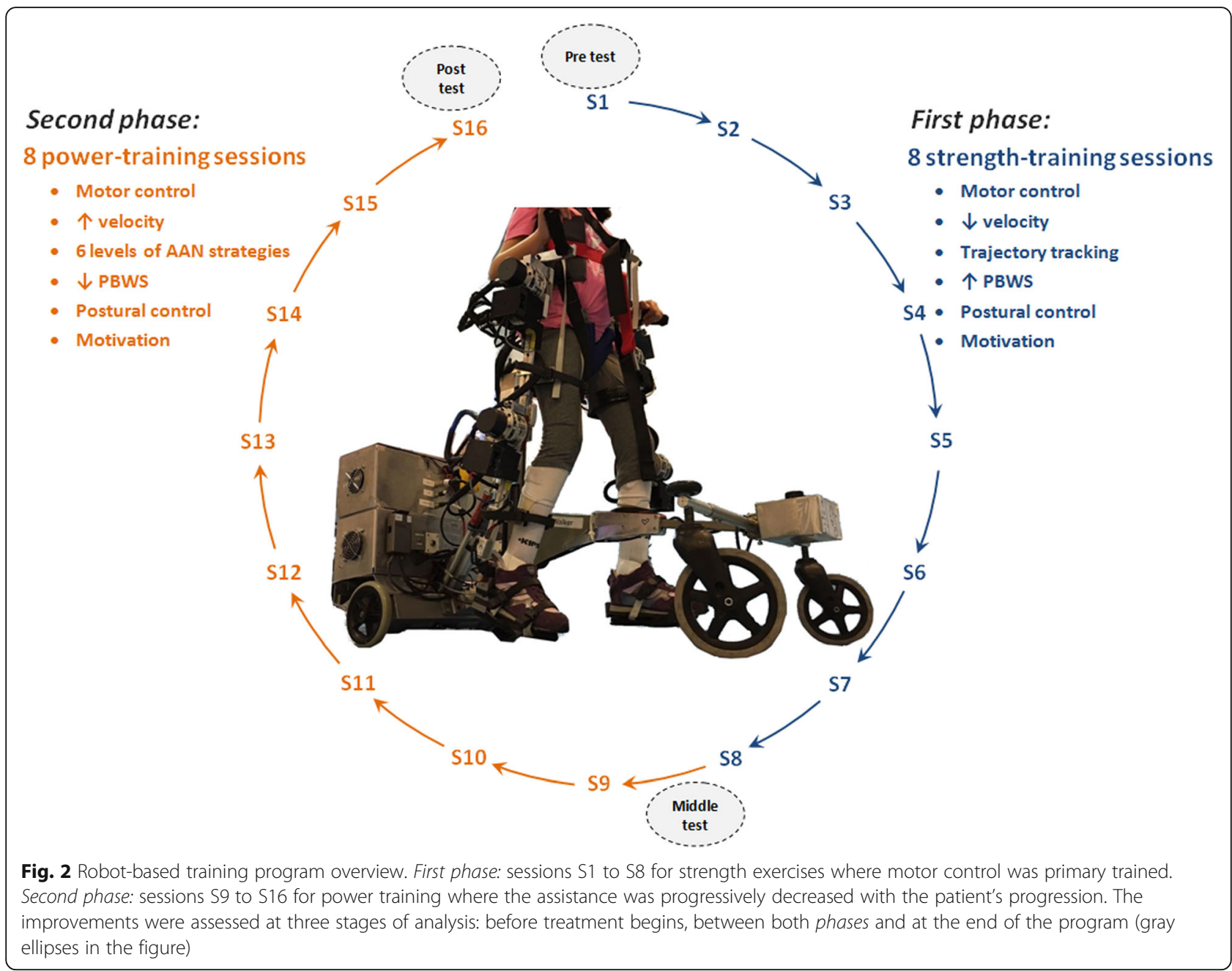

robot imposed a gait trajectory tracking. Sessions 9 to 16 were related to muscle power performance through levels of AAN strategies, where self-activity was required.

\section{Training phases}

In order to individually define the training progression through the different sessions and to comply with the NSCA guidelines, the principal modifications were implemented on ROM, PBWS and gait velocity. The selected parameters for both phases are represented by Figs. 3 and 4 respectively. This selection was concluded in collaboration with our clinical partners, based on the evaluation of previous studies carried out with CPWalker [9, 18]. The robot-based tasks began with high assistance and PBWS, and they progressed toward greater ROM and smaller PBWS as long as the patient overcame the different levels of the sessions. Parameter variations within and between sessions were performed as long as session goals were attained and when the clinical staff agreed, based on levels of spasticity, fatigue and motor control presented in the last day. If the patient

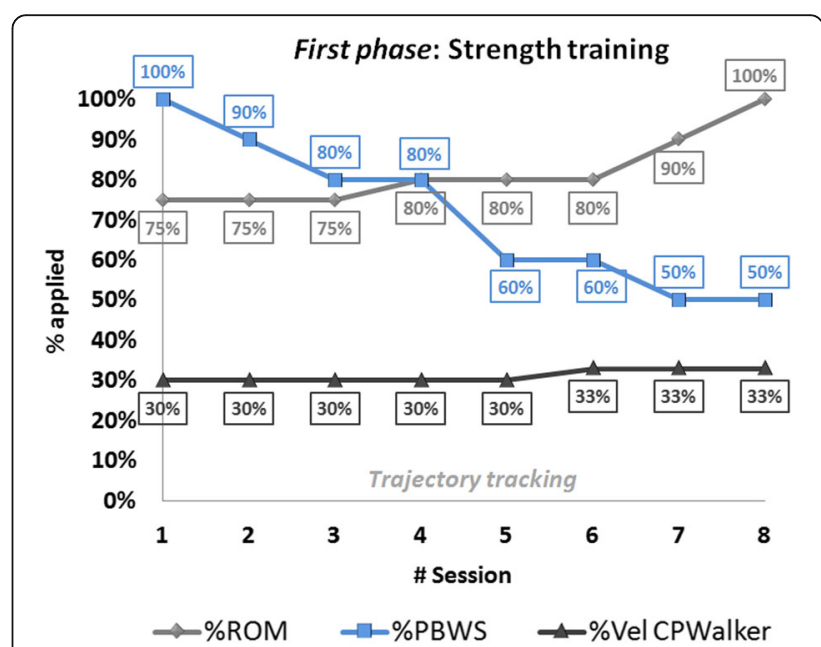

Fig. 3 First phase: strength training progression values along first 8 sessions of the robot-based therapy. Trajectory tracking motion was imposed by the robot. The light-gray line represents the movement amplitude (\%ROM), the blue line the changes of \%PBWS and the dark-gray line is referred to gait velocity percentage for each session 


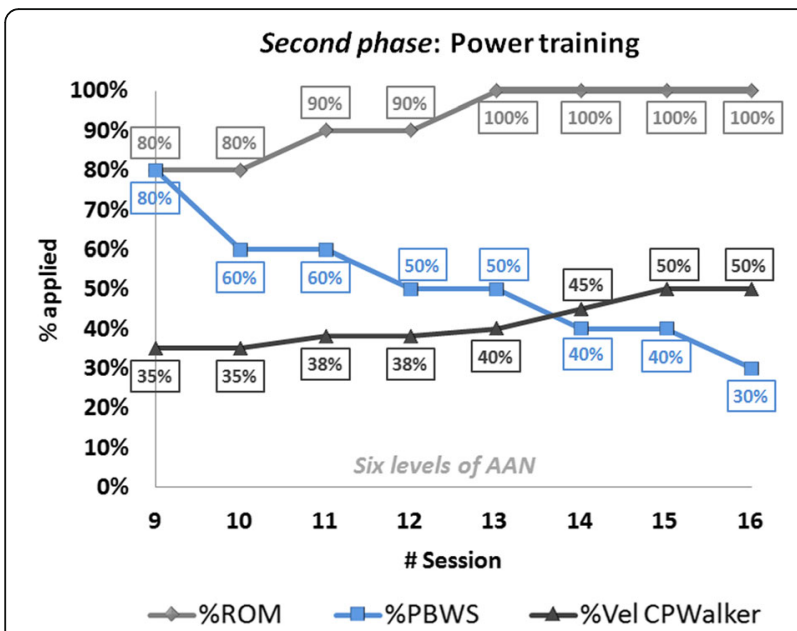

Fig. 4 Second phase: power training progression values along sessions 9 to 16 of the robot-based therapy. Six different levels of assistance were selected on the exoskeleton (combining hips and knees). The light-gray line represents the movement amplitude (\%ROM), the blue line the changes of \%PBWS and the dark-gray line is referred to gait velocity percentage for each session

was not ready to jump to the next challenge, the session was repeated with the same last percentages.

Within the first phase (Fig. 3), the first session was performed with the children completely suspended $(100 \%$ of PBWS) in order to adapt the users to the movements with the robot. Moreover, during random moments of this first session, the patients wore a mask on their eyes to feel the motion performance. For the rest of the first phase children's lower limbs were guided through a pure position control imposed by the CPWalker platform, with a gradual decrease in PBWS (Fig. 3 blue), and a gradual amplification of ROM (Fig. 3 light-grey). Gait velocity during this first phase was maintained around a regular and small value (Fig. 3 dark-grey). Notice that in general, only one variable at each session was varied.

The second phase of the training (sessions 9 to 16 in Fig. 4) presented an additional difficulty that enhanced the user's collaboration in the exercises performance through different levels of AAN strategies in the exoskeleton. The initial ROM for the second phase was set at $80 \%$ of the total gait pattern and reached $100 \%$ by session 13 (Fig. 4 light-grey), time at which velocity was highly increased (Fig. 4 dark-grey). Note that gait velocity for this phase became around double of the one achieved in the first phase, which is in relation to the requisites exposed in Table 2. Furthermore, PBWS declined up to $30 \%$ of weight supported by the platform (Fig. 4 blue).

It is important to highlight in Figs. 3 and 4 that the percentage of PBWS was related to the individual patient's total weight, and the percentage of ROM was applied to the total trajectory of the gait pattern programmed in the control of CPWalker [7]. The estimated changes in gait velocity are represented regarding percentages of CPWalker platform, where $100 \%$ corresponded to $0.6 \mathrm{~m} / \mathrm{s}$.

\section{Tailored assist as needed strategies}

With the aim of enhancing the patient's participation in the second phase and consequently improving outcomes of the treatment, in sessions 9 to 16, position control was substituted by six adapted levels of impedance control in the joints of the exoskeleton (Table 3).

One of the main advantages of CPWalker robot was the possibility of selecting three different modes of assistance individualized per joint beyond a pure position control (high, medium and low impedances). In consonance with this, six situations (levels) were adopted following the criteria of our clinical partners in order to define the scales of difficulty in the assistance of knees and hips in the second phase (Table 3). Thereby, the patients were considered fit to move to the next level when they achieved a performance higher than $85 \%$ in the execution of each session, together with its corresponding parameters (ROM, PBWS and gait velocity) represented by Fig. 4. This percentage of performance was calculated comparing the real motion executed by the children and the desired gait pattern of each session.

\section{Postural control}

It was important to ensure that throughout all the sessions, patients maintained a proper posture of head and trunk because it facilitates the performance of any activity of daily living, and improves the social interaction, the participation and communication $[12,19]$. In this sense, the aim of our proposal was to provide biofeedback to the patients each time that they kept an incorrect position of the body during walking. With this goal, the CPWalker robot used Inertial Measurement Units (IMUs) (Technaid, Spain) to measure the rotation of head and trunk in real time, and give acoustic feedback when subjects overcame predefined maximum values selected by clinicians. In response to the acoustic feedback, patients were instructed to correct their position, time at which the acoustic feedback ceased. This strategy had been previously proved with promising results in children with spastic diplegia [9].

Table 3 Levels of assistance in first and second training phases

\begin{tabular}{|c|c|c|c|c|c|c|c|}
\hline & \multirow{2}{*}{$\begin{array}{l}\text { First phase } \\
\text { Level } 0\end{array}$} & \multicolumn{6}{|c|}{ Second phase } \\
\hline & & Level 1 & Level 2 & Level 3 & Level 4 & Level 5 & Level 6 \\
\hline Hips & $P$ & $\mathrm{HI}$ & $\mathrm{MI}$ & $\mathrm{Ml}$ & $\mathrm{MI}$ & LI & $\mathrm{LI}$ \\
\hline Knees & $P$ & $P$ & $P$ & $\mathrm{HI}$ & $\mathrm{Ml}$ & $\mathrm{Ml}$ & $\mathrm{LI}$ \\
\hline
\end{tabular}

The assistance on knee joint always went behind the assistance on hip, due to the knee movement during gait is performed following inertial forces, assigning to the hip movement higher importance. A higher level is implemented if the session performance (real motion versus desired pattern) is bigger than $85 \%$

$P$ position, $H I$ high impedance, $M I$ medium impedance, $L I$ low impedance 


\section{Motivation and inclusion of challenges}

In the field of physical rehabilitation, especially in childhood disability, the F-words (Function, Family, Fitness, Fun, Friends and Future) defined by Dr. Rosenbaum [20] become really important. It is essential to maintain a high patients' motivation because this concept could affect treatment outcomes [21]. To address this issue, we introduced challenges with goals in each session of the robot-based therapy with the aim of having a more engaged user. An example of that is a classification board where the children could follow their progression along the different sessions, and they were rewarded when the goals of each session were correctly fulfilled.

Moreover, with the same objective of enhancing motivation, the data collected with the robotic platform was explained to the patients through graphics so they could feel part of the team, and their interest in the treatment increased.

The patient's motivation was subjectively measured in each session through a scale from 0 to 10 points, with 0 being no motivation and 10 being maximum motivation.

\section{Metrics}

In order to objectively measure the patient's evolution and due to the lack of homogeneity among children with $\mathrm{CP}$, we decided to evaluate the progression of the therapy by comparing each patient to himself, instead of maintaining a control group. We carried out some analyzes and evaluation metrics in different occasions of the study (Table 4): during the use of the robot, before the treatment begins (pre), in the middle and after the whole sessions (post).

The 10mwt was assessed for two situations: normal comfortable walking speed and maximum walking speed. Three trials were collected for each situation and subsequently, the average of the three trials was calculated.

Regarding the 6mwt, it was performed indoors along a flat corridor, where the walking course had a $30 \mathrm{~m}$ length and was marked every $3 \mathrm{~m}$. The turnaround points were marked with cones. The patients received information about remaining time every minute, but they were not encouraged during the exercise [22]. The heart rate was also measured for each patient in two situations: resting and just after finishing the test. This parameter gives the possibility of calculating the Physiological Cost Index (PCI) after the exercise, which is used to quantify the energy expended by the patients during the exercise and their progression, [23].

3D gait analysis was recorded at $200 \mathrm{~Hz}$ using a motion capture system Smart-DX (BTS Bioengineering, Italy). In order to obtain gait kinetics, a set of reflective markers were placed over the skin on discrete anatomical sites according to the Helen Hayes Model [24]. Subjects walked barefoot at a self-selected speed.

Maximum isometric strength was measured in $\mathrm{kgf}$ with a hand-held dynamometer microFET2 (Hoggan Scientific LLC, USA). Three records were taken and averaged for each movement bilaterally (dorsiflexion, plantarflexion, knee flexion-extension, hip flexion-extension, abduction and adduction).

Table 4 Evaluation metrics and moment of application

\begin{tabular}{|c|c|c|c|c|c|}
\hline \multirow[t]{2}{*}{ Metric } & \multirow[t]{2}{*}{ Utility } & \multicolumn{4}{|l|}{ When } \\
\hline & & During & Pre & Middle & Post \\
\hline 10-m walking test (10 mwt) [30] & Gait-speed measure & & * & * & * \\
\hline 6-min walking test (6 mwt) [33] & $\begin{array}{l}\text { Global responses involved during exercise. } \\
\text { Walked distance and endurance }\end{array}$ & & * & * & * \\
\hline Physiological Cost Index [23] & Energy expended & & & * & * \\
\hline 3D gait analysis & $\begin{array}{l}\text { Kinematics, spatial-temporal parameters, } \\
\text { gait deviation index (GDI), gait profile score (GPS)... }\end{array}$ & & * & * & * \\
\hline $\begin{array}{l}\text { Maximum isometric strength with } \\
\text { hand-held dynamometer }\end{array}$ & Maximum voluntary contraction of primary lower limbs muscles & & * & * & * \\
\hline $\begin{array}{l}\text { Selective Control Assessment of } \\
\text { Lower Extremity (SCALE) [25] }\end{array}$ & Quantify selective voluntary motor control and its progression & & * & * & * \\
\hline $\begin{array}{l}\text { Gross Motor Function Measure (GMFM-88) } \\
\text { dimensions D (standing) and E (walking) [26] }\end{array}$ & $\begin{array}{l}\text { Evaluate changes in gross motor function according to } \\
\text { activities as walking, running or jumping }\end{array}$ & & * & & * \\
\hline Kinesiophobia assessment & Psychological influence of fear and pain & & * & & * \\
\hline $\begin{array}{l}\text { Gillette Functional Assessment } \\
\text { Questionnaire (FAQ) [34] }\end{array}$ & Independent activities, locomotor abilities and user's satisfaction & & * & & * \\
\hline ROM performance & Measure of ROM in lower limbs during robot walking & * & & & \\
\hline Selective force & Evolution of forces executed by the user during robot walking & * & & & \\
\hline Patient's motivation & Estimation of user's motivation in 0 to 10 scale & * & & & \\
\hline
\end{tabular}


The particularity of the SCALE assessment [25] was that it was evaluated by the same physiotherapist bilaterally on three occasions (pre, middle and post), with the aim of reducing the subjective error.

The changes of GMFM-88 [26] were collected for the 88 items, but we focused on dimensions D (standing) and $\mathrm{E}$ (walking).

The kinesiophobia assessment consisted of a test composed of 10 questions of 1 to 4 points each. The responses were given by the patients without parents' influence.

Two FAQ questionnaires were requested: one as initial questionnaire at the beginning and the other as follow up at the end of the treatment. These surveys presented several questions for parents and others referred to children.

During the whole treatment, ROM performance and force interactions were measured for each session in order to evaluate if the patient was prepared to jump to the next stage with more difficult parameters and level of assistance.

Finally, the users' motivation was subjectively evaluated by the practitioner from 0 to 10 points for each session with the robot.

\section{Patients}

Four children diagnosed with spastic CP affecting muscle strength and motor control of lower limbs (two male, two female, weight $44.75 \pm 6.29 \mathrm{~kg}$, height $1.56 \pm$ $0.29 \mathrm{~m}$ and age $14.50 \pm 2.38$ years-old) were selected to be participants for testing the robotic training proposal (P1, P2, P3 and P4 in Table 5). The inclusion criteria for patients' recruitment followed: i) children aged 11 to 18 years suffering from spastic diplegia; ii) Gross Motor Function Classification System (GMFCS [1]) levels I to IV; iii) maximum weight $75 \mathrm{~kg}$; iv) anthropometric measures of lower limbs according to the exoskeleton of CPWalker; v) capable of understanding the proposed exercises; and vi) able to signal pain or discomfort. The exclusion criteria was: i) patients who experimented concomitant treatments 3-months prior study (e.g. orthopedic surgery or botulinum toxin); ii) children with muscle-skeletal deformities or unhealed skin lesions in the lower limbs that could prevent the use of

Table 5 Patients' description

\begin{tabular}{|c|c|c|c|c|c|c|}
\hline \multirow[t]{2}{*}{ Patient } & \multirow[t]{2}{*}{ Age } & \multirow[t]{2}{*}{ GMFCS } & \multirow{2}{*}{$\begin{array}{l}\text { Weight } \\
(\mathrm{kg})\end{array}$} & \multirow{2}{*}{$\begin{array}{l}\text { Height } \\
\text { (m) }\end{array}$} & \multicolumn{2}{|c|}{ Walking support } \\
\hline & & & & & $50 \mathrm{~m}$ & $500 \mathrm{~m}$ \\
\hline P1 (F) & 12 & III & 40 & 1.56 & $\mathrm{CT}$ & WC \\
\hline P2 (F) & 16 & $\|$ & 42 & 1.60 & Cane & Cane \\
\hline P3 (M) & 17 & III & 54 & 1.53 & PW & WC \\
\hline P4 (M) & 13 & $\|$ & 43 & 1.55 & - & - \\
\hline
\end{tabular}

Two females (F) and two males (M) with spastic diplegia were selected. No medication 3-months prior the study was taken by the patients. The type of walking support without the aid of the robot is indicated for a distance of $50 \mathrm{~m}$ and $500 \mathrm{~m}$ : crutches-CT, wheelchair-WC, posterior walker-PW and cane the exoskeleton; iii) patients with critical alterations of motor control as dystonia, choreoathetosis or ataxia; iv) aggressive or self-harming behaviors; and v) severe cognitive impairment. The study was carried out at "Hospital Infantil Universitario Niño Jesús", (Spain). The Local Ethical Committee of this hospital gave approval to the study (R-0032/12) and warranted its accordance with the Declaration of Helsinki. All participants and families were informed, and parental consents were obtained prior to participation (Additional file 1).

\section{Results}

Due to personal reasons unrelated to the study, three of four patients (P1, P2 and P4) completed 15 of a possible 16 total sessions. Concretely, P1 lost the session number 8 , P2 lost the number 7 and P4 the number 11 . The rest of training was completed successfully.

The modifications of parameters (ROM, PBWS and gait velocity) proposed in Figs. 3 and 4, were fulfilled by all children without problems. The progressions of the levels of assistance provided by Table 3, which were tailored for each patient between sessions 9 to 16 (power training with AAN strategies), are represented in Fig. 5, where the maximum reached level was level 5 by P1 in the last two sessions.

\section{Gait speed, endurance and global responses}

All patients improved the outcomes in D and E dimensions (assessed together) of the GMFM-88 scale [26] (Fig. 6a). Showing the dimensions D and E separately, the increments per patient (post-pre) were: P1 $\left(38.48 \%_{\mathrm{D}}\right.$, $\left.34.71 \%_{\mathrm{E}}\right)$, P2 $\left(5.13 \%_{\mathrm{D}},-1.39 \%_{\mathrm{E}}\right)$, P3 $\left(25.90 \%_{\mathrm{D}}, 15.72 \%_{\mathrm{E}}\right)$ and P4 $\left(10.26 \%_{\mathrm{D}}, 22.20 \%_{\mathrm{E}}\right)$. Regarding these results, all patients except P2 got minimal clinically important difference (MCID) for dimensions D and E [27]. The rest of dimensions of the GMFM-88 showed in conjunction a total increment for this scale of $18.16 \%$ in $\mathrm{P} 1,3.93 \%$ in P2, 11.8\% in P3 and 8.11\% in P4, which represent for P1, P3 and P4 gains higher than seven percentage points, considered as positive changes by therapists.

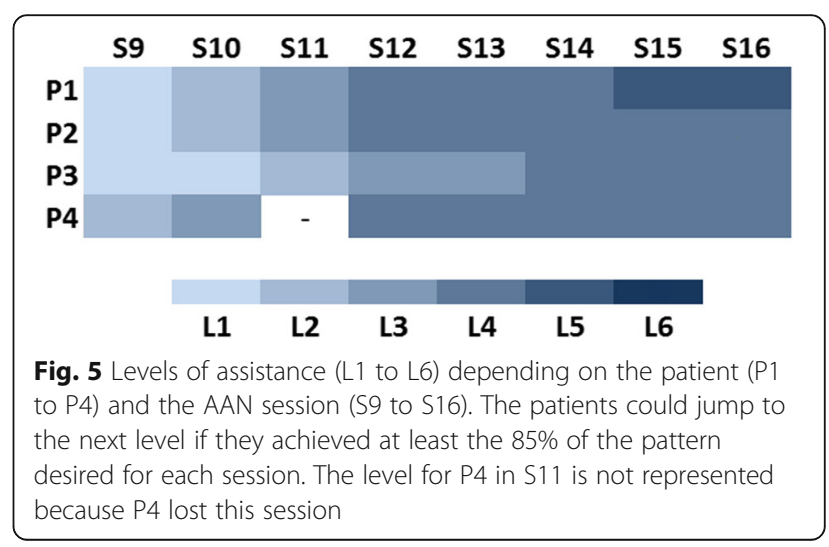




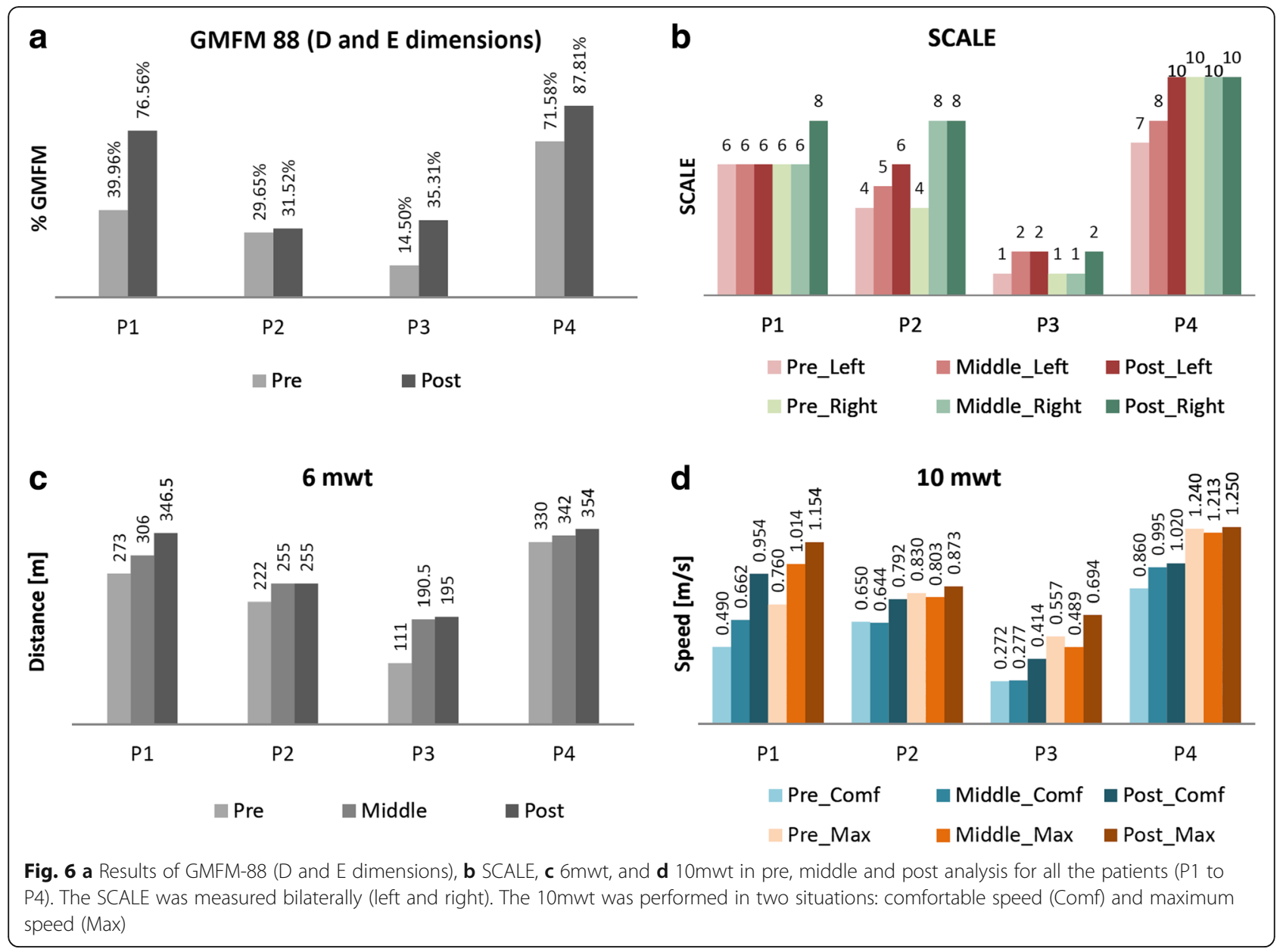

The SCALE assessment also showed better results at the end of the robot-based treatment (Fig. 6b), [25]. In this case, although the value for the left leg in P1 was kept same as at the beginning (Fig. 6b, red bars for P1), the rest of measures were increased or maintained as maximum (SCALE equal to 10 points). No reference of MCID has been found for SCALE assessment.

Finally, both the walked distance in the 6mwt and the walking speed in the 10mwt increased after the training period (Fig. 6c and d respectively). The two situations evaluated for the 10mwt are represented: a comfortable speed for each child (blue bars in Fig. 6d) and the same exercise at maximum speed (orange bars in Fig. 6d). More concretely, the percentage of progressions comparing post and pre-analysis in these metrics, were: P1 (6mwt: $26.92 \%$; 10mwt $\mathrm{momf}_{\text {f }}$ 94.69\%; 10mwt $\mathrm{max}_{\text {max }}$ :51.84\%); P2 (6mwt: 14.86\%; 10mwt comf $_{1}$ 21.85\%; 10 mwt $_{\max }$ : 5.18\%); P3 (6mwt: $75.68 \%$; 10 mwt $_{\text {comf }}$ : 52.21\%; $\left.10 \mathrm{mwt}_{\max }: 24.60 \%\right)$ and P4 (6mwt: $7.27 \% ; 10 \mathrm{mwt}_{\mathrm{comf}}$ : $18.60 \%$; 10 mwt $\left._{\text {max }}: 0.81 \%\right)$.

In case of $6 \mathrm{mwt}$, the MCID was established as $50 \mathrm{~m}$ in stroke survivors [28]. In children and adolescents with $\mathrm{CP}$, the minimum detectable change (MDC), which may not represent a MCID, has been settled as $64 \mathrm{~m}$ for GMFCS II and $47.4 \mathrm{~m}$ for GMFCS III [29]. Comparing our results to these significances, $\mathrm{P} 1$ and $\mathrm{P} 3$ reached the MCID for the $6 \mathrm{mwt}$ with improvements of $73.5 \mathrm{~m}$ and $84 \mathrm{~m}$ respectively.

In case of $10 \mathrm{mwt}, 0.13 \mathrm{~m} / \mathrm{s}$ is the MCID in spinal injuries [30]. For CP, only the MDC was found, but Thompson et al. ensured in [29] that it did not present reliability. In that sense, P1 and P3 obtained clinical significance in the $10 \mathrm{mwt}_{\max }$ comparing with the MCID exposed in [30].

The Physiological Cost Index was evaluated comparing middle and post assessments during the 6mwt. All patients reduced the PCI: P1 obtained 0.75 beats $/ \mathrm{m}$ (middle) and 0.55 beats $/ \mathrm{m}$ (post); P2 0.89 beats $/ \mathrm{m}$ (middle) and 0.80 beats $/ \mathrm{m}$ (post); P3 1.57 beats $/ \mathrm{m}$ (middle) and 1.26 beats $/ \mathrm{m}$ (post); and P4 0.33 beats $/ \mathrm{m}$ (middle) and 0.03 beats $/ \mathrm{m}$ (post).

\section{Strength progression}

In order to quantify the patient's maximum strength performing on defined and individual movements without the robot, three measures were taken for each required motion. According to that, Fig. 7 represents the average 


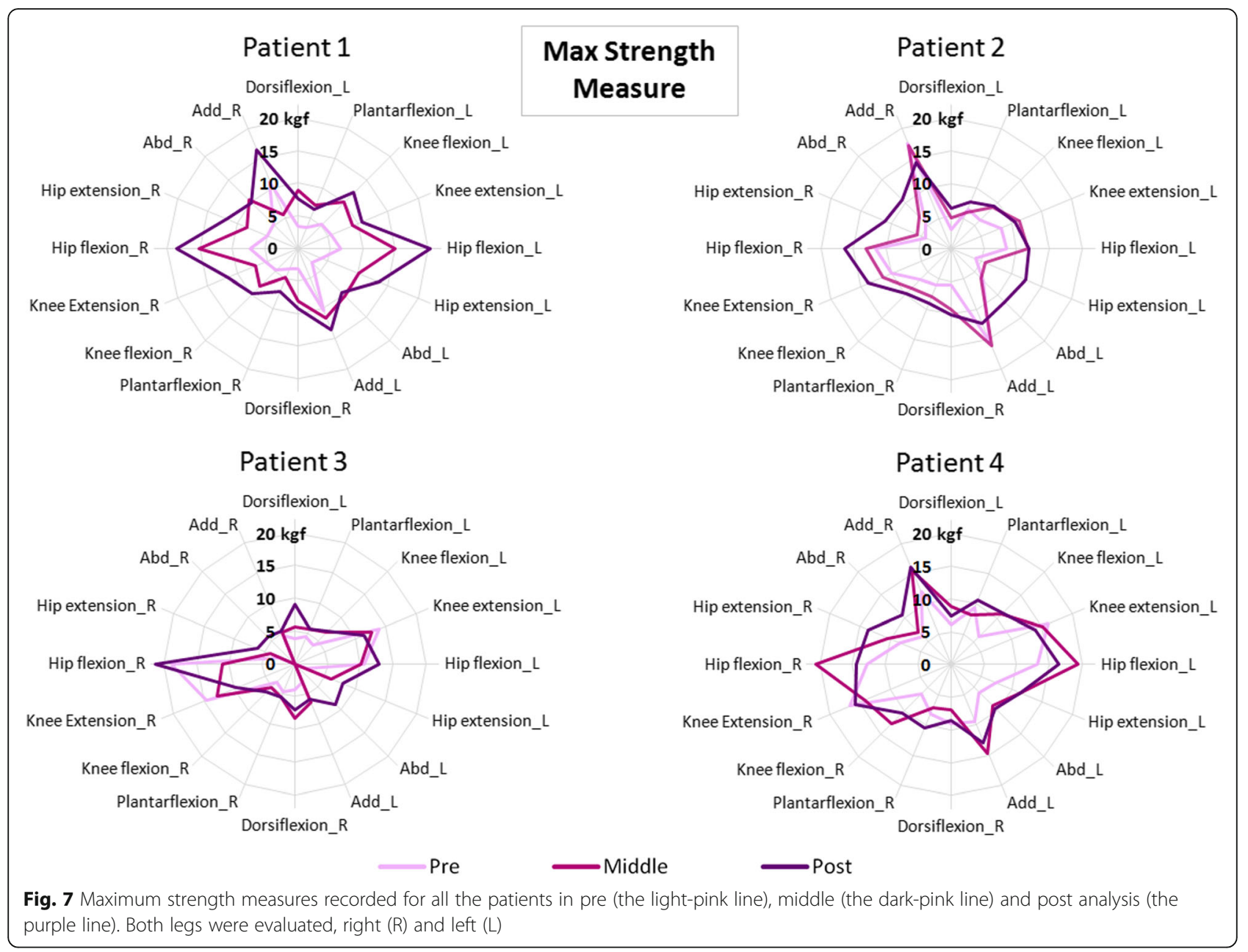

values (in kgf) of the individualized movements recorded in pre, medium and post analyzes. In general, results from Fig. 7 show that the purple line (post assessment) covers the light-pink line (pre measures) for all the patients. In some cases, it even covers the dark-pink line (measured just after finishing the first 8 strength training sessions). This means that higher values of strength were reached by the children after the robot-based treatment. Concretely, the general improvements (including all the required movements) per patient in maximum isometric strength measure, comparing pre and post averages, were: P1: $129.77 \pm 58.71 \%$; P2: $61.39 \pm 58.55 \%$; P3: 70.54 $\pm 83.68 \%$ and $\mathrm{P} 4: 34.41 \pm 30.41 \%$.

\section{Kinematics and spatiotemporal variability}

The 3D kinematic analysis provided outcomes focused on gait improvements respect to normality. The GPS and GDI (Fig. 8) are accepted indexes that represent how close the patient's gait is to the desired gait. Related to these metrics and comparing pre and post analyzes, all the patients obtained better values for both sides (left and right) after the robot-based treatment. Nevertheless, they were not clinically significant, except for the right side of P1 (around 10 points in GDI). It is important to highlight that we believe that the post results in P4 could be affected by personal circumstances non-related to the study that occurred the day of the test.

Table 6 shows the values of Fig. 8 in detail and also includes some of the spatial-temporal parameters recorded during the studies. The average improvement percentages (four patients) in spatiotemporal parameters were: $21.46 \pm 33.79 \%$ for mean velocity, $2.84 \pm 13.96 \%$ for cadence and $17.95 \pm 20.45 \%$ for step length.

\section{ROM performance}

Although all the patients succeeded the changes in the parameters of ROM, velocity and PBWS for the different sessions, the progression in AAN levels was individualized for each subject (Fig. 5). Thereby, P1 was the most advanced, achieving level 5 as the maximum. An example of ROM performance difference between trajectory tracking and AAN strategy is represented by Fig. 9, which shows the data collected for P1 in session 3 (Fig. 9a) and session 


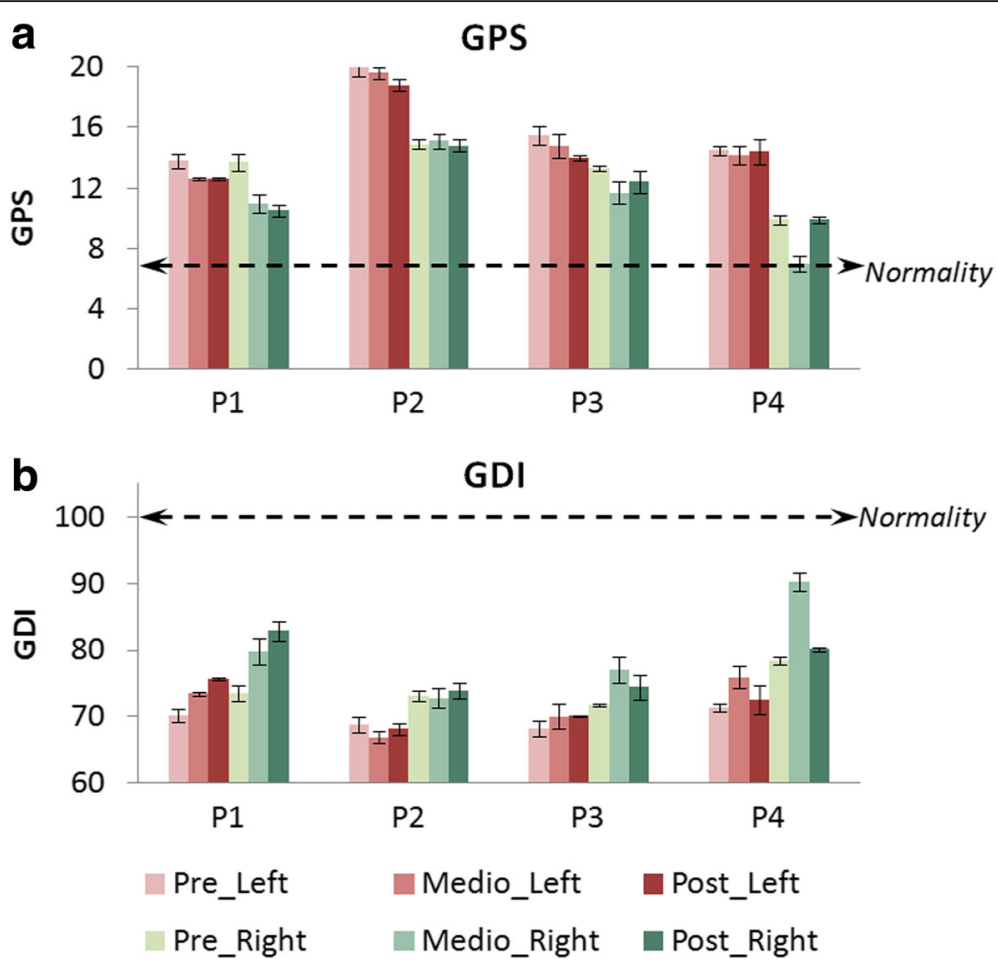

Fig. 8 a GPS and $\mathbf{b}$ GDI for pre, middle and post kinematic analyzes of patients P1 to P4. The results represent means \pm standard error bilaterally (left in red bars and right in green bars). Normality in GPS considers values lower than 7 points (doted-black line in (a)), and normality in GDI comprehends values higher than 100 points (doted-black line in (b))

16 (Fig. 9b). The last one was recorded within level 5 (low impedance at hips and medium impedance at knees).

\section{Qualitative variables}

The motivation was subjectively evaluated by the practitioner who was with the children during the whole period of the study. The averaged motivation values on a scale from 0 to 10 points were: 9.4 for P1, 8.6 for P2, 9.44 for P3 and 8.87 for P4. Moreover, three of four patients decreased the kinesiophobia score after the 16 sessions.

Parents and patients filled a FAQ questionnaire at the beginning and a follow-up at the end of the treatment. Regarding parent questionnaires, results show that all of them thought that the strength and mobility were better at the end of the study thanks to the robot-based therapy. Meanwhile half of them also included the endurance as an improved variable due to the robot. One-hundred percent of parents felt satisfied towards the results of robotic therapy with CPWalker, and they ensured that they would like to do it again. The patients' opinion was very similar. They were also satisfied and, in general, they described the treatment as: "really fun", "the robot makes you feel light and independent" and "safe".

\section{Discussion and Conclusion}

The main aim of this research was to provide a first approach to the implementation of a novel and defined robotic rehabilitation method that could cover the most important clinical aspects of the ICF-CY framework. This proposal was tested with four pediatric patients with $\mathrm{CP}$, which provided us some preliminary outcomes to assess it. Although the patients' progression was evaluated without a control group, we do not consider it as a relevant limitation of the study, since the wide variety of differences among each child with $\mathrm{CP}$ makes interesting and even necessary to expose the improvements by comparing each patient with himself.

According to the results, the greatest benefits due to the robot-based treatment corresponded to P1 and P3, who were the most affected levels of GMFCS (III in both cases). In general, the higher values of gait speed and improved values of global responses achieved by all the children in several tests, may be in benefit of the patients' social mobility. Visual inspection of the graphics show that changes appeared after a small number of sessions (middle tests) and they were commonly increased or maintained until the post studies. The results showed statistical significance for the improvements of most of 
Table 6 Spatial-temporal parameters, GPS and GDI values for pre, middle and post analyzes in all the patients. The results were calculated taking an average of 40 steps

\begin{tabular}{|c|c|c|c|c|c|c|c|c|}
\hline Patient & Analysis & Side & Stride time (s) & $\begin{array}{l}\text { Mean velocity } \\
(\mathrm{m} / \mathrm{s})\end{array}$ & $\begin{array}{l}\text { Cadence } \\
\text { (steps/min) }\end{array}$ & Step length (m) & $\begin{array}{l}\text { Gait Profile Score } \\
\text { (GPS) }\end{array}$ & $\begin{array}{l}\text { Gait Deviation Index } \\
\text { (GDI) }\end{array}$ \\
\hline Normality & & & $0.93 \pm 0.04$ & $1.20 \pm 0.20$ & $129.60 \pm 8.40$ & $0.58 \pm 0.06$ & $<7$ & $>100$ \\
\hline \multirow[t]{6}{*}{ P1 } & Pre & Left & $1.52 \pm 0.12$ & $0.50 \pm 0.1$ & $79.05 \pm 7.51$ & $0.39 \pm 0.02$ & $13.80 \pm 0.50$ & $70.19 \pm 1$ \\
\hline & & Right & $1.54 \pm 0.15$ & & & $0.38 \pm 0.04$ & $13.70 \pm 0.60$ & $73.50 \pm 1.13$ \\
\hline & Middle & Left & $1.81 \pm 0.04$ & $0.40 \pm 0$ & $66.20 \pm 1.58$ & $0.25 \pm 0.18$ & $12.60 \pm 0.10$ & $73.43 \pm 0.30$ \\
\hline & & Right & $1.82 \pm 0.12$ & & & $0.40 \pm 0.03$ & $11 \pm 0.60$ & $79.82 \pm 2$ \\
\hline & Post & Left & $1.27 \pm 0$ & $0.80 \pm 0$ & $96 \pm 1.30$ & $0.46 \pm 0.04$ & $12.60 \pm 0.10$ & $75.62 \pm 0.16$ \\
\hline & & Right & $1.23 \pm 0.03$ & & & $0.56 \pm 0.02$ & $10.50 \pm 0.40$ & $82.87 \pm 1.42$ \\
\hline \multirow[t]{6}{*}{ P2 } & Pre & Left & $1.42 \pm 0.04$ & $0.50 \pm 0$ & $86.70 \pm 5.19$ & $0.26 \pm 0.01$ & $20 \pm 0.60$ & $68.81 \pm 1.23$ \\
\hline & & Right & $1.36 \pm 0.11$ & & & $0.39 \pm 0.01$ & $14.90 \pm 0.30$ & $73.08 \pm 0.78$ \\
\hline & Middle & Left & $1.43 \pm 0.08$ & $0.40 \pm 0$ & $86.80 \pm 1.86$ & $0.22 \pm 0.02$ & $19.60 \pm 0.40$ & $66.93 \pm 0.84$ \\
\hline & & Right & $1.34 \pm 0.04$ & & & $0.39 \pm 0.01$ & $15.10 \pm 0.50$ & $72.83 \pm 1.41$ \\
\hline & Post & Left & $1.58 \pm 0.14$ & $0.40 \pm 0.10$ & $76 \pm 7.50$ & $0.25 \pm 0.04$ & $18.80 \pm 0.40$ & $68.12 \pm 0.87$ \\
\hline & & Right & $1.61 \pm 0.18$ & & & $0.41 \pm 0$ & $14.80 \pm 0.40$ & $73.96 \pm 1.21$ \\
\hline \multirow[t]{6}{*}{ P3 } & Pre & Left & $1.33 \pm 0.27$ & $0.30 \pm 0$ & $84.90 \pm 9.90$ & $0.27 \pm 0.01$ & $15.50 \pm 0.60$ & $68.23 \pm 1.19$ \\
\hline & & Right & $1.58 \pm 0.03$ & & & $0.15 \pm 0.03$ & $13.30 \pm 0.20$ & $71.67 \pm 0.20$ \\
\hline & Middle & Left & $1.73 \pm 0.23$ & $0.20 \pm 0.10$ & $66.20 \pm 8.23$ & $0.17 \pm 0.12$ & $14.80 \pm 0.80$ & $70.08 \pm 1.91$ \\
\hline & & Right & $1.98 \pm 0.25$ & & & $0.12 \pm 0.04$ & $11.70 \pm 0.70$ & $77.08 \pm 2.03$ \\
\hline & Post & Left & $1.43 \pm 0.08$ & $0.40 \pm 0$ & $86.80 \pm 4.45$ & $0.39 \pm 0.01$ & $14 \pm 0.20$ & $70.11 \pm 0.16$ \\
\hline & & Right & $1.35 \pm 0.08$ & & & $0.14 \pm 0.10$ & $12.40 \pm 0.70$ & $74.46 \pm 1.88$ \\
\hline \multirow[t]{6}{*}{ P4 } & Pre & Left & $1.02 \pm 0.06$ & $0.80 \pm 0$ & $121.20 \pm 7.12$ & $0.41 \pm 0.03$ & $14.50 \pm 0.30$ & $71.43 \pm 0.57$ \\
\hline & & Right & $0.97 \pm 0.06$ & & & $0.37 \pm 0.02$ & $9.90 \pm 0.30$ & $78.39 \pm 0.63$ \\
\hline & Middle & Left & $1 \pm 0.05$ & $0.90 \pm 0$ & $121.35 \pm 4.48$ & $0.44 \pm 0.01$ & $14.20 \pm 0.60$ & $75.94 \pm 1.70$ \\
\hline & & Right & $0.99 \pm 0.03$ & & & $0.44 \pm 0.01$ & $7 \pm 0.50$ & $90.23 \pm 1.42$ \\
\hline & Post & Left & $0.96 \pm 0.02$ & $0.90 \pm 0$ & $121.20 \pm 1.80$ & $0.46 \pm 0.03$ & $14.40 \pm 0.80$ & $72.59 \pm 2.17$ \\
\hline & & Right & $1.02 \pm 0.01$ & & & $0.47 \pm 0$ & $9.90 \pm 0.20$ & $80.13 \pm 0.33$ \\
\hline
\end{tabular}

the tests, reaching also clinical significance in some of them, especially for P1 and P3.

The most challenging part was the second phase of the training, which allowed the possibility of adapting the level of assistance depending on the patient's progression. Thereby, any subject could achieve the last level (level 6), and although the action of reaching level 4 took P3 longer than the rest of children, this patient could pass through it in the last three sessions.

It is interesting to highlight that the outcomes from isometric strength measure showed important peaks of improvement, especially for hip and knee flexion-extension, which was targeted with the CPWalker robotic platform. These higher values were observed from the middle to the post analysis. The results of the present study are difficult to compare with the scientific literature due to the lack of studies using exoskeletons for gait resistance training. However, we can establish that our results are in line with previous studies assessing conventional resistance strength training in CP [31].
In relation to 3D-kinematic analysis, as we said before, P4 suffered a non-grata personal situation the day of the post study. We believe this affected the results of this metric. Nevertheless, the whole population improved the values for spatiotemporal parameters, GDI and GPS, although some of these improvements were not clinically significant. It is important to highlight that not only the kinematics was improved, also the physiological expenditure in walking activities decreased, as illustrated by the reduction of the PCI in all patients, which means that better gait performance implies lower energy cost.

Finally, the motivation scale for the patients and the parents' satisfaction with the robot-based treatment was very high in most aspects.

The greatest achievements of this proposal come from the possibility of exercising different gait functions in an orderly way, individualized per joint and at the same time than over-ground walking. The proposed protocol could be applied to any current robotic device for gait rehabilitation making minimal changes on it: e.g. in 


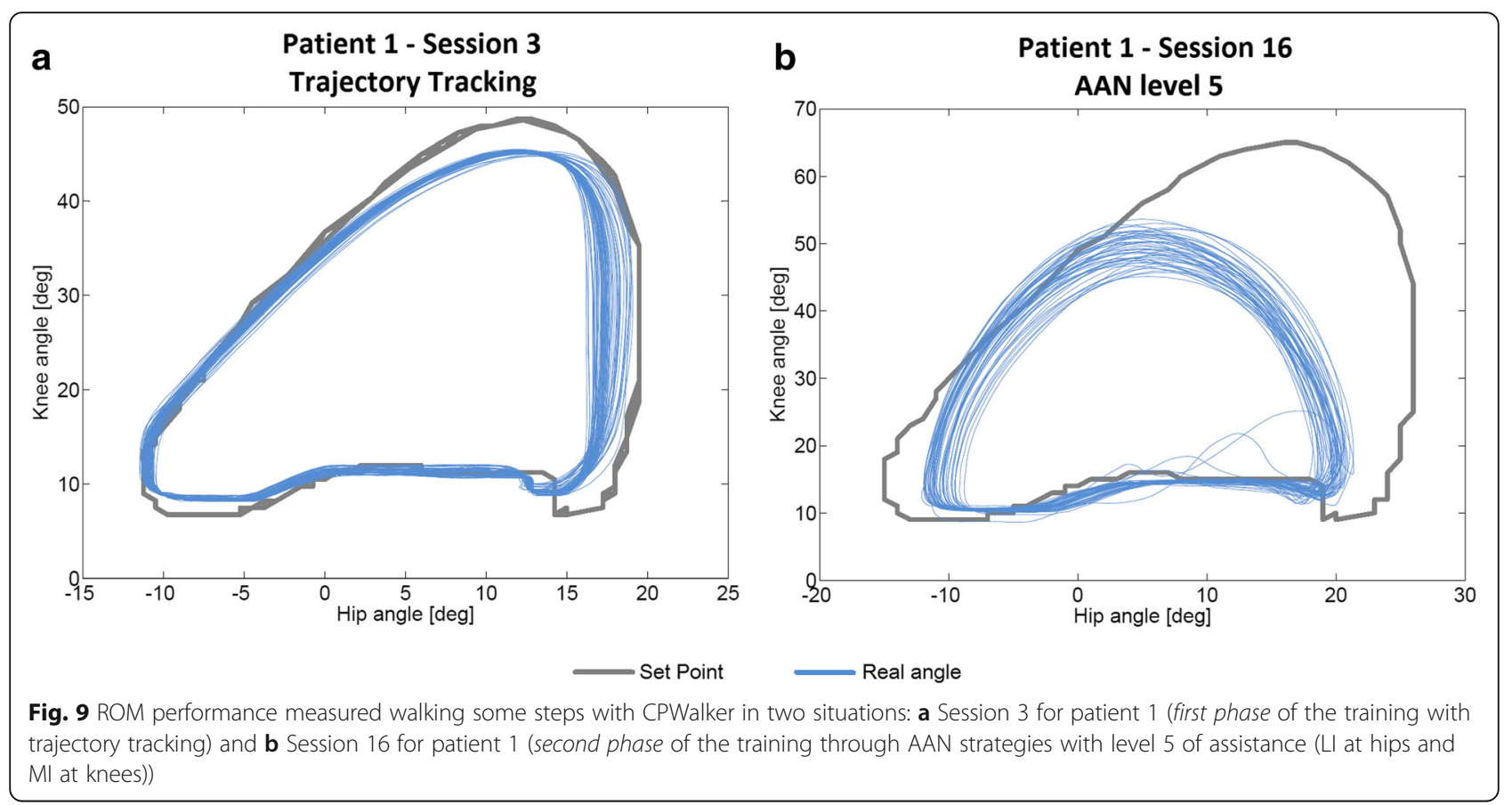

treadmill pediatric platforms as Lokomat [14], despite the impossibility of over-ground walking, it already has different controllers, whose operation modes are close to the levels of impedance of CPWalker, ensuring the progression of the therapy into the sessions. Regarding working postural control in parallel with lower limbs training, which we considered one of the key factors of the study, it may be solved through other solutions if the selected robotic device does not have a similar strategy as described with CPWalker, but it is crucial to guarantee its compliance to get the best results of the treatment [12].

The principal limitation of this research is that we followed up only in short term, so further research with a higher population size is needed to evaluate if the improvements will be kept over time. Moreover, although other studies propose interventions on 3 non-consecutive days per week [31], the patients of the present study performed the robotic exercises during 2 non-consecutive sessions per week. This enabled them to continue their conventional therapies in parallel to the robot-based rehabilitation. The conventional therapies had been attended on a regular basis for years, so we considered to not abolish them for ethical reasons. The conventional therapies of the patients were performed 2 days a week and consisted of exercises on balance and strength focusing on quadriceps and abs. Although the patients were doing conventional and robotic therapy in parallel, we consider that the improvements achieved in this proposal are exclusively associated to the use of the CPWalker, since the patients received usual care therapies for years with no significant improvements in their functional situation. In conclusion, the method implemented with CPWalker is complementary to the common therapies, providing new possibilities to the clinical practice through robotic rehabilitation and also reaching better outcomes than conventional therapy alone.

We are currently working on new future lines in which we plan to include electromyography during the use of the robotic trainer in order to assess more objectively the patients' progressions. Electromyography data will serve to evaluate the therapy outcomes in terms of reorganization of the neural structures, which will be performed by analyzing the raw neural drive to muscles and the muscle synergies.

In a nutshell, this manuscript contributed with a defined robotic treatment that could be implemented in most of the existing rehabilitation robotic devices for lower limbs, and which we evaluated positively in four patients with $\mathrm{CP}$ using $\mathrm{CPW}$ alker.

\section{Additional file}

Additional file 1: Implementation of the proposal and patients' progression during robotic training. (MP4 $2330 \mathrm{~kb}$ )

\section{Abbreviations}

10mwt: 10 m walking test; 6 mwt: 6 min walking test; AAN: Assist as needed; CNS: Central nervous system; CP: Cerebral Palsy; FAQ: Functional Assessment Questionnaire; GDI: Gait deviation index; GMFCS: Gross motor function classification system; GMFM: Gross motor function measure; GPS: Gait profile score; ICF-CY: International Classification of Functioning, Disability and Health framework, Children and Youth version; IMUs: Inertial measurement units; MCID: Minimal clinically important difference; MDC: Minimal detectable change; NSCA: National Strength and Conditioning Association; PBWS: Partial 
body weight support; PCl: Physiological cost index; ROM: Range of motion; SCALE: Selective control assessment of lower extremity

\section{Acknowledgements}

Authors would like to thank Biomechanical Institute of Valencia and other colleges from Spanish National Research Council and Niño Jesús Hospital for their collaboration in other aspects and achievements carried out with CPWalker robotic platform. Authors appreciate Dr. Gaebler's advice for the development of the robotic program proposal.

We would like to thank Made for Movement company for supporting us with NF-Walker device.

We also acknowledge the effort and contribution from all testing children and their families.

We acknowledge support of the publication fee by the CSIC Open Access Publication Support Initiative through its Unit of Information Resources for Research (URICI).

\section{Funding}

The work presented in this paper has been carried out with the financial support from the Ministerio de Economía y Competitividad of Spain, under Contract DPI2012-39133-C03-01.

\section{Availability of data and materials}

The data supporting the conclusions of this article are included within the article and its additional files.

\section{Authors' contributions}

$\mathrm{CB}$ collaborated in the design of the training program, developed the control algorithm of the robotic device, processed the clinical data and drafted the manuscript. TM collaborated in the design of training program proposal and reviewed the manuscript. BM collaborated in the clinical processing data derived from the study and helped with the recording of clinical metrics. OR was involved in the development of the hardware of the robotic device. APS participated in patients' recruitment and collaborated in the recording of clinical metrics. SLL participated in patients' recruitment and collaborated in the review and critique of the manuscript. IM aided in the patient's recruitment and reviewed and criticized the manuscript. ER collaborated in all facets of the contribution. All authors read and approved the final manuscript.

\section{Ethics approval and consent to participate}

The Local Ethical Committee of the "Hospital Infantil Universitario Niño Jesús" gave approval to the study and warranted its accordance with the Declaration of Helsinki. The study was carried out with the number R-0032/ 12 from Local Ethical Committee of the Hospital, and was publicly registered with the number ISRCTN18254257 as clinical trial. All patients and families were informed beforehand and provided consent through parents to participate.

\section{Consent for publication}

Consent for publication has been given by parents or legal guardians of involved patients.

\section{Competing interests}

The authors declare that they have no competing interests.

\section{Publisher's Note}

Springer Nature remains neutral with regard to jurisdictional claims in published maps and institutional affiliations.

\section{Author details}

${ }^{1}$ Centre for Automation and Robotics (CAR), CSIC-UPM, Ctra Campo Real km 0.2 - La Poveda-Arganda del Rey, 28500 Madrid, Spain. ${ }^{2}$ Hospital Infantil Universitario Niño Jesús, Madrid, Spain. ${ }^{3}$ Universidad Rey Juan Carlos, Madrid, Spain. ${ }^{4}$ Universidad de La Salle, Madrid, Spain. ${ }^{5}$ Universidade Federal do Espírito Santo Vitória, Brazil.
Received: 4 April 2017 Accepted: 10 July 2018

Published online: 27 July 2018

\section{References}

1. Palisano R, Rosenbaum P, Walter S, Russell D, Wood E, Galuppi B. Development and reliability of a system to classify gross motor function in children with cerebral palsy. Dev Med Child Neurol. 1997;39:214-23. PMID: 9183258.

2. Dietz V. Clinical aspects for the application of robotics in locomotor neurorehabilitation. In: Reinkensmeyer DJ, Dietz V, editors. Neurorehabilitation Technol: Springer; 2016. p. 209-21. https://doi.org/10. 1007/978-3-319-28603-7_11.

3. Meyer-Heim A, a van Hedel HJ. Robot-assisted and computer-enhanced therapies for children with cerebral palsy: current state and clinical implementation. Semin Pediatr Neurol. 2013;20:139-45. https://doi.org/10. 1016/j.spen.2013.06.006

4. Bayón C, Raya R, Lara SL, Ramírez Ó, Serrano J, Rocon E. Robotic therapies for children with cerebral palsy: a systematic review. Transl Biomed. 2016;7: 1-10. https://doi.org/10.21767/2172-0479.100044.

5. Diaz I, Gil J, Sanchez E. Lower-limb robotic rehabilitation: literature review and challenges, J robot. 2011.

6. van Asseldonk EHF, van der Kooij H. Robot-Aided Gait Training with LOPES. In: Dietz V, editor. Neurorehabilitation Technol. London: Springer London; 2012. p. 379-96. https://doi.org/10.1007/978-1-4471-2277-7_21.

7. Bayón C, Ramírez O, Serrano Jl, Del Castillo MD, Pérez-Somarriba A, Belda-Lois JM, Martínez-Caballero I, Lerma-Lara S, Cifuentes C, Frizera A, Rocon E. Development and evaluation of a novel robotic platform for gait rehabilitation in patients with cerebral palsy: CP Walker. Robotics Autonomous Systems. 2017;91:101-14. https://doi.org/10.1016/j.robot.2016.12.015.

8. Bartenbach V, Gort M, Riener R. Concept and Design of a Modular Lower Limb Exoskeleton. In: 6th IEEE RAS/EMBS Int. Conf. Biomed. Robot. Biomechatronics; 2016. p. 649-54. https://doi.org/10.1109/BIOROB.2016.7523699.

9. Bayón C, Lerma S, Ramírez O, Serrano Jl, Del Castillo MD, Raya R, Belda-Lois JM, Martínez I, Rocon E. Locomotor training through a novel robotic platform for gait rehabilitation in pediatric population: short report. J Neuroeng. Rehabil. 2016;13:98. https://doi.org/10.1186/s12984-016-0206-X.

10. Rüdt S, Moos M, Seppey S, Riener R, Marchal-Crespo L. Towards more efficient robotic gait training: a novel controller to modulate movement errors, 6th IEEE RAS/EMBS Int. Conf Biomed Robot Biomechatronics; 2016. p. 884-9.

11. Lefmann S, Russo R, Hillier $S$. The effectiveness of robotic-assisted gait training for paediatric gait disorders: systematic review. J Neuroeng Rehabil. 2017;14:1. https://doi.org/10.1186/s12984-016-0214-X.

12. Wallard L, Dietrich G, Kerlirzin Y, Bredin J. Robotic-assisted gait training improves walking abilities in diplegic children with cerebral palsy. Eur J Paediatr Neurol. 2017:1-8. https://doi.org/10.1016/j.ejpn.2017.01.012.

13. World Health Organization. International classification of functioning, disability and health, children and youth version: World Health Organization; 2007.

14. Aurich-Schuler T, Grob F, Van Hedel HJA, Labruyère R. Can Lokomat therapy with children and adolescents be improved? An adaptive clinical pilot trial comparing guidance force, path control, and FreeD. J Neuroeng Rehabil. 2017;14:1-14. https://doi.org/10.1186/s12984-017-0287-1.

15. Hussain S, Xie SQ, Liu G. Robot assisted treadmill training : mechanisms and training strategies. Med Eng Phys. 2011;33:527-33. https://doi.org/10.1016/j. medengphy.2010.12.010

16. Van Asseldonk EHF, Veneman JF, Ekkelenkamp R, Buurke JH, Van Der Helm FCT, Van Der Kooij $H$. The effects on kinematics and muscle activity of walking in a robotic gait trainer during zero-force control. IEEE Trans Neural Syst Rehabil Eng. 2008;16:360-70. https://doi.org/10. 1109/TNSRE.2008.925074.

17. Damiano DL, Abel MF. Functional outcomes for strenght training in spastic cerebral palsy. Arch Phys Med Rehabil. 1998;79:119-25.

18. Martín-Lorenzo T, Lerma-Lara S, Bayón C, Ramírez Ó, Rocon E. The CPWalker for strength training in children with spastic cerebral palsy: a training program proposal. In: Int. Conf Neurorehabilitation; 2016.

19. Dewar $\mathrm{R}$, Love $\mathrm{S}$, Johnston LM. Exercise interventions improve postural control in children with cerebral palsy: a systematic review. Dev Med Child Neurol. 2014; https://doi.org/10.1111/dmcn.12660.

20. Rosenbaum P, Gorter JW. The "F-words" in childhood disability: I swear this is how we should think! Child Care Health Dev. 2012;38:457-63. https://doi. org/10.1111/j.1365-2214.2011.01338.x. 
21. Maclean N, Pound P. A critical review of the concept of patient motivation in the literature on physical rehabilitation. Soc Sci Med. 2000;50:495-506. PMID: 10641802. http://www.ncbi.nlm.nih.gov/pubmed/10641802. Accessed 11 Dec 2016.

22. Borg G. Psychophysical bases of perceived exertion. Med Sci Sport Exerc. 1982;14:377-81.

23. Butler P, Engelbrecht M, Major RE, Tait JH, Stallard J, Patrick JH. Physiological cost index of walking for normal children and its use as an Indicator of physical handicap. Dev Med Child Neurol. 1984;26:607-12. https://doi.org/ 10.1111/j.1469-8749.1984.tb04499.x.

24. Kadaba MP, Ramakrishnan HK, Wootten ME. Measurement of lower extremity kinematics during level walking. J Orthop Res. 1990;8:383-92. http://trama.deib.polimi.it/allegati/Kadaba_1990.pdf (Accessed 22 Dec 2015)

25. Fowler EG, Staudt LA, Greenberg MB, Oppenheim WL. Selective control assessment of the lower extremity (SCALE): development, validation, and interrater reliability of a clinical tool for patients with cerebral palsy. Dev Med Child Neurol. 2009;51:607-14. https://doi.org/10.1111/j.1469-8749.2008.03186.x.

26. D.J. Russell, P.L. Rosenbaum, L.M. Avery, M. Lane, Gross motor function measure (GMFM-66 and GMFM-88) user's manual, press, Cambridge University, 2002.

27. Kaphingst KA, Persky S, Lachance C. Outcome tools used for ambulatory children with cerebral palsy: responsiveness and minimum clinically important differences. Dev Med Child Neurol. 2008;50:918-25. https://doi. org/10.1080/10810730902873927.Testing.

28. Rehabilitation Measures Database, 6 Minute Walk Test, (n.d.). https://www. rehabmeasures.org (Accessed 7 May 2018).

29. Thompson P, Beath T, Bell J, Jacobson G, Phair T, Salbach NM, Wright VF. Test-retest reliability of the 10-metre fast walk test and 6-minute walk test in ambulatory school-aged children with cerebral palsy. Dev Med Child Neurol. 2008;50:370-6. https://doi.org/10.1111/j.1469-8749.2008.02048.x.

30. Rehabilitation Measures Database, Timed 10-Meter Walk Test, Timed 10-m. Walk TEST (n.d.). www.rehabmeasures.org (Accessed 11 Aug 2016).

31. Park EY, Kim WH. Meta-analysis of the effect of strengthening interventions in individuals with cerebral palsy. Res Dev Disabil. 2014;35:239-49. https:// doi.org/10.1016/j.ridd.2013.10.021.

32. Faigenbaum C, Kramer AD, Blimkie W. Youth resistance training: updated position statement paper from the National Strength and conditioning association. J Strength Con Res. 2009;23:60-79. https://doi.org/10.1519/JSC. Ob013e31819df407.

33. Crapo RO, Casaburi R, Coates AL, Enright PL, Maclntyre NR, McKay RT, Johnson D, Wanger JS, Zeballos RJ, Bittner V, Mottram C. ATS statement: guidelines for the six-minute walk test. Am J Respir Crit Care Med. 2002;166: 111-7. https://doi.org/10.1164/rccm.166/1/111.

34. Gorton GE, Stout IL, Bagley AM, Bevans K, Novacheck TF, Tucker CA. Gillette functional assessment questionnaire 22-item skill set: factor and Rasch analyses. Dev Med Child Neurol. 2011;53:250-5. https://doi.org/10.1111/j. 1469-8749.2010.03832.x.

Ready to submit your research? Choose BMC and benefit from:

- fast, convenient online submission

- thorough peer review by experienced researchers in your field

- rapid publication on acceptance

- support for research data, including large and complex data types

- gold Open Access which fosters wider collaboration and increased citations

- maximum visibility for your research: over $100 \mathrm{M}$ website views per year

At BMC, research is always in progress.

Learn more biomedcentral.com/submissions 